\title{
The impact of epidemic information on the public's worries and attitude toward epidemic prevention measures during the COVID-19 outbreak
}

\author{
Hai-Ping Liao ${ }^{1}$ (D) - Jin-Liang Wang ${ }^{1}$ (D) \\ Accepted: 13 January 2021 / Published online: 28 January 2021 \\ (C) The Author(s), under exclusive licence to Springer Science+Business Media, LLC part of Springer Nature 2021
}

\begin{abstract}
Since the outbreak of 2019 coronavirus disease (COVID-19) in December 2019, the Chinese government has implemented effective epidemic prevention measures. To provide useful information for governments to manage this public health crisis, we conducted an online survey among Chinese general population from February 24 to 28, 2020. In this study, we examined the impact of epidemic information and rumors on public's worries and attitude toward prevention measures during the outbreak of COVID-19. A total of 853 valid questionnaires (641 women, 75.1\%) were collected from 24 provincial regions in China. Most respondents' ages ranged from 18 to 60 (833 participants, 97.66\%). A mediation model was built to analyze the influence of epidemic information and rumors on worries and attitude. The results showed that the amount of epidemic information positively predicted public's worries, which in turn predicted a supportive attitude toward the prevention measures. Worries partially mediated the relationship between the amount of epidemic information and the supportive attitude. The amount of rumors negatively predicted the supportive attitude. The results of this study implied the importance of timely and credible information providing to evoke a certain level of worry and promote public cooperation, and the necessary attention to refute and resist rumors for effective risk communication in a public health crisis.
\end{abstract}

Keywords COVID-19 $\cdot$ Epidemic information $\cdot$ Rumor $\cdot$ Worry $\cdot$ Attitude

\section{Introduction}

Since the outbreak of COVID-19 epidemic in December, 2019, this disease has caused serious health and economic damages and induced a wide range of public worries. To cope with the epidemic, effective epidemic prevention measures have been implemented throughout China. Literatures have suggested a supportive attitude is imperative to ensure the effectiveness of these measures (Sibley et al., 2020; Smith, 2006). Furthermore, the duration of the epidemic has exceeded public's expectation, making public's supportive attitude more indispensable. Given that public's psychological and behavioral response are often based on the information they acquire (Yamashita, 2012), this study aimed to investigate the impact of epidemic information on

Jin-Liang Wang

wangjinliang09@gmail.com

1 Center for Mental Health Education, Faculty of Psychology, Southwest University, Chongqing, No. 2 Tiansheng Road, Beibei District, Chongqing 400715, China public's worries and attitude toward prevention measures during the outbreak of COVID-19 epidemic (in February 2020, before the WHO declared it a pandemic). An understanding on these relations would shed light on the mechanism via which supportive attitude toward prevention measures is formed during the pandemic, and would provide guidance on a successful implementation of the prevention.

\section{Epidemic Information and Worries about COVID-19}

The COVID-19 epidemic has attracted worldwide attention, and public were drowning in a mass of epidemic information including content about the fast spreading of the virus, the ongoing prevention measures, the death and damages caused by this disease. Public's psychological responses can be affected by these information (Dong \& Zheng, 2020; Yamashita, 2012). Extensive research has found that epidemic information increased individuals' perception of risk (Khosravi, 2020; Olagoke, Olagoke, \& Hughes, 2020), and thus caused a lot of worries (Brooks et al., 2020; Dong \& 
Zheng, 2020). Specifically, during the COVID-19 outbreak, individuals may worry about themselves or their family members getting infected, have concerns about the lack of protective equipment and medical resources, and dread possible family financial issues as well as regional economic problems. Worry is an emotional state stimulated by the anticipation of a negative outcome and uncertainty about the future, and a reaction to an individual's cognitive assessment of risk (Borkovec, Ray, \& Stöber, 1998; Kummeneje \& Rundmo, 2019). Therefore, because epidemic information leads to an increase of perceived risk, the amount of epidemic information would be positively associated with public's worries about COVID-19 (Hypothesis 1).

\section{Epidemic Information and Attitude Toward Epidemic Prevention Measures}

To fight with the damaging epidemic, China has been implementing effective epidemic prevention measures. However, such measures would not be effective without the public's support attitude and compliance(Sibley et al., 2020; Smith, 2006; Wallace, Paulson, Lord, \& Bond, 2005). Importantly, public's attitude may depend on epidemic information they acquire(Yamashita, 2012). During or after disasters such as an epidemic, when useful information about epidemic as well as its coping strategies are provided, individuals tend to exhibit more affinity or voluntary behavior, leading to public's effective and adaptive collective actions (Glik, 2007; Greenaway $\&$ Cruwys, 2019). Furthermore, epidemic information may promote public risk communication through timeously updating the updated information about epidemic. Effective risk communication in public health crisis can increase subjective risk and motivate people to willingly cooperate with management measures (Glik, 2007; Vaughan \& Tinker, 2009). Epidemic information is essential in risk communication because epidemic information conveys risk information and behavior guidance for public to motivate appropriate self-protective behavior (Vaughan \& Tinker, 2009). Hence, the amount of epidemic information may positively predict public's supportive attitude toward prevention measures (Hypothesis 2).

In addition, epidemic information may indirectly promote public support for epidemic prevention measures by arousing the feeling of worry. During the risk communication process, epidemic information as warnings demands attention, stimulate memory, evoke emotion, and communicate consequences and safe behavior (Buck \& Ferrer, 2012). As an emotional response to the threat of epidemic, worry may be the psychological precondition for epidemic information to guide the public to adopt officially recommended prevention measures during COVID-19. Furthermore, worry is also an aversive motivational state. Worry may motivate individuals encountering a threat to prepare for possible negative outcomes and evoke their defensive avoidance response to the threat (Mogg $\&$ Bradley, 2004), such as staying at home to avoid infection. Therefore, epidemic information might make people more supportive of prevention measures and encourage them to take protective behavior through evoking a feeling of worry. Hence, it is possible that worries over the pandemic promote the supportive attitude (Hypothesis 3 ) and play a mediating role between epidemic information and public's supportive attitude (Hypothesis 4).

It is important to clarify that the present study examined the public's explicit and not implicit attitude toward the measures. The Associative-Propositional Evaluation Model (APE) posits that an explicit attitude is formed in a propositional process, which is controlled by consciousness and social rules (Gawronski \& Bodenhausen, 2006, 2007). Negative emotion promotes the propositional process of attitude formation (Gawronski \& Bodenhausen, 2006; Wu, Fang, Sun, \& Li, 2013). Hence, although epidemic prevention measures cause inconvenience and cost, out of compliance with social rules, individuals may form a supportive explicit attitude after a propositional process because these measures can effectively control the epidemic, especially when they feel worried.

\section{Rumors among Epidemic Information}

Despite the possibility that epidemic information may promote more supportive attitude, it is worth noting that not every piece of epidemic information is conductive to risk communication. Among the wide variety of epidemic information, rumors are rampant and have been blamed for failures of risk communication (Garrett, 2001; Glik, 2007; Tasnim, Hossain, \& Mazumder, 2020). Rumors threaten the public's confidence and cooperative attitude during public health crises (Ali, 2020; Jalali \& Mohammadi, 2020), because rumors always cause misunderstandings and panic, especially in the internet era where information spreading with few costs (Parikh et al., 2020). Therefore, rumors may cause a failure of risk communication and damage the public's supportive attitude toward epidemic prevention measures during the COVID-19 outbreak (Hypothesis 5).

Rumors may also evoke worry. Although no studies have revealed a direct relationship between rumors and worry, research on the relationship between rumors and anxiety has shed light on the matter. Rumors may stimulate anxiety in recipients of rumor transmission (Rosnow, 1980). This is because widespread rumors during epidemic usually contain issues of great importance for a group and appear ambiguous (Rosnow, 1980), and individuals are more likely to be anxious over issues of importance and ambiguity (Freeston, Rhéaume, Letarte, Dugas, \& Ladouceur, 1994). As a cognitive component of anxiety (Borkovec et al., 1998), worry is likely to increase when rumors are spread. Despite this, it is also possible that worry will encourage individuals to take epidemic 
prevention measures to protect themselves. Accordingly, rumors may indirectly promote individuals' support for epidemic prevention measures via inducing more worries. Consequently, there may be two opposite processes involved in the influence of rumors on attitude toward prevention measures. In other words, rumors may lead to unsupportive attitude toward prevention measures (Hypothesis 6), while indirectly improve supportive attitude by the mediating role of worries (Hypothesis 7). This is an inconsistent mediation model (MacKinnon, Fairchild, \& Fritz, 2007) wherein indirect effect may weaken the impact of direct effect.

It's also found that being older, female, and more educated is associated with a higher chance of adopting the behaviors suggested by authorities during pandemics (Bish \& Michie, 2010). Economic status also plays a role in that those facing financial problems due to an epidemic may be unwilling to follow epidemic prevention measures (Khosravi, 2020; Vaughan \& Tinker, 2009). Consequently, demographic factors, such as gender, age, income, and educational level, were employed as control variables in this study. In accordance with the considerations noted, this study develops a hypothesis model of the relationships between epidemic information, rumors, worry, and attitude toward COVID-19 (Fig. 1).

\section{Method}

\section{Participants and Procedure}

To examine the relationship between epidemic information, worry and attitude toward epidemic prevention measures, a cross-sectional online questionnaire survey was conducted from February 24 to 28, 2020 in China, and permission was obtained from Southwest University's Human Research Ethics Committee. All participants started their online questionnaire by signing an informed consent. After answering all the questionnaire items, they submitted the answered questionnaire and got their pay of $5 ¥$. We collected 903 questionnaires, and after excluding repetitive and uncompleted questionnaires, a total of 853 valid questionnaires (641 women, $75.10 \%$ ) could be analyzed, thus yielding a callback rate of $94.46 \%$. The participants came from 24 provincial regions, including Sichuan (311 respondents, $36.46 \%$ ) and Chongqing (284 respondents,

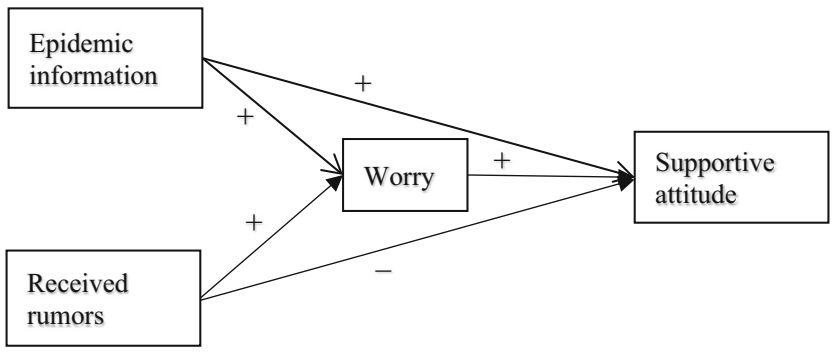

Fig. 1 The hypothesis model
$33.29 \%$ ), and most of them aged from 18 to 60 (833 respondents, $97.66 \%$ ). There were no confirmed or suspected cases of COVID-19 among the respondents. And none of them were close-contacts. The demographic characteristics were demonstrated in Table 1 in detail.

\section{Measures}

\section{The Amount of Epidemic Information}

Through a single question in which respondents were asked how often they usually browse information about COVID-19 in a day, the frequency of information viewing was measured as a predictor of the amount of epidemic information the public had browsed. The respondents indicated how often they did so on a 5-point Likert scale, ranging from 1 (never) to 5 (always). A higher score indicated a higher frequency of epidemic information browsing and thus a larger amount of epidemic information received.

\section{The Amount of Received Rumors}

Through a single question, the respondents had to indicate the amount of information they had browsed about the epidemic that had been confirmed to be rumors. Although such a method could not fully capture the number of rumors in the epidemic information, it was assumed to reflect the proportion of rumors in the information browsed by the public. This could be regarded as significant for the management and publication of information regarding the epidemic. The participants had to indicate this on a 5-point Likert scale, in which 1-5 represented $\leq 20 \%, 21 \%-40 \%, 41 \%-60 \%, 61 \%-80 \%$, and $81 \%-$ $100 \%$, respectively. Thus, higher scores indicated that they had come across more rumors.

\section{Worry}

The epidemic worry scale comprises seven items assessed on a 5-point scale (Table 2). This scale was developed by the authors to estimate the Chinese public's feelings of worry during COVID-19. The higher the mean score of the seven items, the greater was the feeling of worry. The scale has good reliability and validity: Cronbach's $\alpha=.926, \chi^{2} / d f=2.171, p$ $=.027, \mathrm{CFI}=.998, \mathrm{TLI}=.995, \mathrm{RMSEA}=.037(95 \% \mathrm{CI}=$ $.012, .061)$. All the factor loadings were larger than .695 . More information about the results of exploratory factor analysis (EFA) and confirmatory factor analysis (CFA) of this scale can be found in the appendices.

\section{Supportive Attitude}

The supportive attitude toward epidemic prevention measures scale was developed by the authors to measure the public's 
Table 1 Demographic characteristics

\begin{tabular}{|c|c|c|c|}
\hline \multicolumn{2}{|l|}{ Demographic variables } & \multirow{2}{*}{$\frac{n}{212}$} & \multirow{2}{*}{$\frac{\%}{25.90 \%}$} \\
\hline Gender & male & & \\
\hline & female & 641 & $75.10 \%$ \\
\hline \multirow[t]{7}{*}{ Age } & under 18 & 19 & $2.22 \%$ \\
\hline & $18-25$ & 648 & $75.97 \%$ \\
\hline & $26-30$ & 76 & $8.91 \%$ \\
\hline & $31-40$ & 47 & $5.51 \%$ \\
\hline & $41-50$ & 56 & $6.57 \%$ \\
\hline & $51-60$ & 6 & $0.70 \%$ \\
\hline & over 60 & 1 & $0.12 \%$ \\
\hline \multirow[t]{7}{*}{ Education } & elementary school graduates & 8 & $0.94 \%$ \\
\hline & junior high school students or graduates & 27 & $3.17 \%$ \\
\hline & high school students or graduates & 45 & $5.28 \%$ \\
\hline & college students or graduates & 376 & $44.08 \%$ \\
\hline & undergraduate students or graduates & 282 & $33.60 \%$ \\
\hline & masters & 95 & $11.14 \%$ \\
\hline & doctors & 20 & $2.34 \%$ \\
\hline \multirow[t]{6}{*}{ Income (per month) } & less than 1000 yuan & 68 & $7.97 \%$ \\
\hline & $1000-3000$ & 241 & $28.25 \%$ \\
\hline & $3000-5000$ & 203 & $23.80 \%$ \\
\hline & $5000-8000$ & 102 & $11.96 \%$ \\
\hline & $8000-12,000$ & 102 & $11.96 \%$ \\
\hline & more than 12,000 & 24 & $2.81 \%$ \\
\hline \multirow[t]{2}{*}{ Location } & rural areas & 450 & $52.75 \%$ \\
\hline & cities or towns & 403 & $47.25 \%$ \\
\hline Total & & 853 & $100.00 \%$ \\
\hline
\end{tabular}

attitude toward epidemic prevention measures in China. This scale comprises four items assessed on a 5-point scale (Table 3). The mean score of the four items was calculated to obtain the final score. High scores indicate a more supportive attitude toward prevention measures. The scale has good reliability and validity: Cronbach's $\alpha=.783, \chi^{2} / d f=2.648, p$ $=.104, \mathrm{CFI}=.999, \mathrm{TLI}=.994, \mathrm{RMSEA}=.044(95 \% \mathrm{CI}=$ $.000, .112)$. All the factor loadings were larger than .425 . More information about the results of EFA and CFA of this scale can be found in the appendices.

\section{Results}

\section{Common Method Bias}

Since the measures of variables in the present study were based on self-report, there is a potential threat for common method bias. The results Harman's one-factor test revealed that there was no common method bias in the current study, with the first factor accounting for $34.894 \%$ of the variance (Podsakoff \& Organ, 1986).

Table 2 The epidemic worry scale

\begin{tabular}{|c|c|c|c|c|c|}
\hline \multirow[t]{2}{*}{ Items: I am worried that... } & \multicolumn{5}{|c|}{ Answers (frequency and proportion) } \\
\hline & Not at all & A little & Certainly & Very much & Extremely \\
\hline 1.my family or I will be affected. & $156(18.3 \%)$ & $346(40.6 \%)$ & $199(23.3 \%)$ & $76(8.9 \%)$ & $76(8.9 \%)$ \\
\hline 2. my family members or I will die or get a carryover from this disease. & $223(26.1 \%)$ & $279(32.7 \%)$ & $168(19.7 \%)$ & $83(9.7 \%)$ & $100(11.7 \%)$ \\
\hline 3. my family or I will not get timely and effective treatment. & $281(32.9 \%)$ & $243(28.5 \%)$ & $170(19.9 \%)$ & $68(8 \%)$ & $91(10.7 \%)$ \\
\hline 4. my family or I will not get enough goods or medical materials. & $175(20.5 \%)$ & $243(28.5 \%)$ & $190(22.3 \%)$ & $137(16.1 \%)$ & $108(12.7 \%)$ \\
\hline 5. there will be financial problems during the epidemic. & $187(21.9 \%)$ & $251(29.4 \%)$ & $212(24.9 \%)$ & $112(13.1 \%)$ & $91(10.7 \%)$ \\
\hline 6.the local epidemic is difficult to control and will continue to spread. & $223(26.1 \%)$ & $276(32.4 \%)$ & $183(21.5 \%)$ & $93(10.9 \%)$ & $78(9.1 \%)$ \\
\hline 7. the local economy will be hit hard. & $146(17.1 \%)$ & $303(35.5 \%)$ & $218(25.6 \%)$ & $110(12.9 \%)$ & $76(8.9 \%)$ \\
\hline
\end{tabular}


Table 3 The supportive attitude toward epidemic prevention measures scale

\begin{tabular}{|c|c|c|c|c|c|}
\hline \multirow[t]{2}{*}{ Items } & \multicolumn{5}{|c|}{ Answers (frequency and proportion) } \\
\hline & Highly disagree & Disagree & Neutral & Agree & Highly agree \\
\hline 1. I agree with the local epidemic prevention measures. & $7(0.8 \%)$ & $9(1.1 \%)$ & $119(14 \%)$ & $435(51 \%)$ & $283(33.2 \%)$ \\
\hline 2. I should comply with the epidemic prevention measures. & $0(0 \%)$ & $4(0.5 \%)$ & $36(4.2 \%)$ & $323(37.9 \%)$ & $490(57.4 \%)$ \\
\hline 3. The medical staff is dedicated to protecting the lives and health of us all. & $1(0.1 \%)$ & $3(0.4 \%)$ & $45(5.3 \%)$ & $246(28.8 \%)$ & $558(65.4 \%)$ \\
\hline $\begin{array}{l}\text { 4. Many workers and volunteers are devoted to } \\
\text { protecting the lives and health of us all. }\end{array}$ & $0(0 \%)$ & $2(0.2 \%)$ & $41(4.8 \%)$ & $268(31.4 \%)$ & $542(63.5 \%)$ \\
\hline
\end{tabular}

\section{Descriptive Results}

\section{The Amount of Epidemic Information}

The data revealed that $5(0.6 \%)$ respondents never browsed information, $214(25.1 \%)$ browsed occasionally, $306(35.9 \%)$ browsed sometimes, 294 (34.5\%) browsed frequently, and 34 (4\%) always browsed. Differential analysis indicated that respondents in different age groups differed with regard to the amount of epidemic information they received, $F(5,846)=$ $3.827, p=.002, \eta^{2}=.023$. Participants aged $31-40$ and $41-$ 50 browsed more epidemic information than those aged 18 25 and $26-30, p_{\mathrm{s}}<.05$. Respondents aged $31-50$ paid more attention to the epidemic and browsed information more frequently. Gender, education levels, income, and place of residence did not influence the amount of epidemic information browsed $\left(p_{\mathrm{s}}>.05\right)$.

\section{The Amount of Received Rumors}

The results revealed that while 493 (57.8\%) respondents reported that less than $20 \%$ of the viewed information had been confirmed as rumors, $212(24.9 \%)$ reported $21 \%-40 \%, 85$ (10\%) reported $41 \%-60 \%, 44(5.2 \%)$ reported $61 \%-80 \%$, and $19(2.2 \%)$ reported that more than $81 \%$ of the information had been confirmed to be rumors. Whereas the results revealed that there were rumors spreading, the numbers thereof were limited $(M=1.690, S D=.995)$, thus indicating that the overall epidemic information was rather reliable. No significant difference was found in the demographic variables $\left(p_{\mathrm{s}}>\right.$ $.05)$.

\section{Worry}

The data indicated that the general public exhibited a certain degree of concern about the epidemic $(M=2.530, S D=$ 1.537). The respondents expressed worry about themselves and their family members' infections, death, and medical treatment; shortage of medical resources and finances; the effectiveness of epidemic prevention measures; and the regional economy (Table 2). The results of MANOVA revealed that the level of worry depended on monthly family income, $F(6,846)=2.807, p=.010, \eta^{2}=.024$. Those with a monthly income of 1000-3000 yuan expressed more worry than those with a monthly income of 8000-12,000 yuan ( $p<$ $.001)$ and those whose income was above 20,000 yuan $(p<$ $.05)$. In general, the level of worry decreased with an increase in income. No significant demographic differences were found $\left(p_{\mathrm{s}}>.05\right)$.

\section{Supportive Attitude}

The Chinese public had a fairly supportive attitude toward the epidemic prevention measures $(M=4.46, S D=0.5)$. The results presented in Table 3 indicate that the respondents generally agreed with the local epidemic prevention measures. They demonstrated good willingness to comply with the epidemic prevention measures as well as a high degree of support and appreciation for the frontline medical staff and other workers involved in the prevention of the epidemic. It is noteworthy that $14 \%$ of the respondents had neutral attitudes to local epidemic prevention measures, around 5\% reported neutral attitudes to the other 3 items (item 2, 3, 4), and a few opposed the measures. No significant demographic differences were found $\left(p_{\mathrm{s}}>.05\right)$ Table 4.

\section{The Relationship between Epidemic Information, Psychological Response, and Attitude}

Correlation analysis demonstrated that the amount of epidemic information the respondents browsed was significantly and positively correlated with a feeling of worry $(r=.100, p<.01)$ and supportive attitude toward the epidemic prevention measures $(r=.184, p<.001)$. However, while the number of received rumors was negatively correlated with a supportive attitude $(r=-.101, p<.01)$, a feeling of worry was positively correlated with a supportive attitude $(r=.123, p<.001)$.

Subsequently, in accordance with the hypotheses of this study, a structure equation model was constructed: The amount of epidemic information and number of received 
Table 4 The mean value, standard deviation, and correlations of study variables

\begin{tabular}{|c|c|c|c|c|c|c|}
\hline & $M$ & $S D$ & 1 & 2 & 3 & 4 \\
\hline 1. The amount of epidemic information & 3.160 & .868 & - & & & \\
\hline 2. The amount of received rumors & 1.690 & .995 & .07 & - & & \\
\hline 3. Worry & 2.530 & 1.037 & $.100^{* *}$ & .040 & - & \\
\hline 4. Supportive attitude & 4.461 & .502 & $.184^{* * * *}$ & $-.101^{* *}$ & $.123^{* * * *}$ & - \\
\hline
\end{tabular}

rumors were independent variables, worry was an intermediary variable, supportive attitude toward the epidemic prevention measures was the dependent variable, and age and income were both converted into sequential variables as control variables to predict worry and supportive attitude. Because the path loadings of age were not significant $\left(p_{\mathrm{s}}>.05\right)$ and the number of rumors failed to predict worry significantly $(\beta=$ $.031, p<.364)$, to simplify the model, these insignificant paths were deleted. The corrected model fit was acceptable (Fig. 2), $\chi^{2} / d f=2.651, p=.031, \mathrm{CFI}=.932, \mathrm{TLI}=.830, \mathrm{IFI}=.936$, RMSEA $=.044(95 \% \mathrm{CI}=.012, .077)$. The amount of epidemic information positively predicted a feeling of worry $(\beta=$ $.084, p=.013)$ and a supportive attitude $(\beta=.186, p<.001)$ toward epidemic prevention measures. The feeling of worry positively predicted support for epidemic prevention measures $(\beta=.130, p<.001)$. Though the number of rumors failed to predict worry as expected $(\beta=.031, p<.364)$, it negatively predicted a supportive attitude toward the epidemic prevention measures $(\beta=-.113, p<.001)$. The mediation analysis showed that worry partially mediated the effect of epidemic information on a supportive attitude toward epidemic prevention measures. The indirect effect was $.011(95 \% \mathrm{CI}=.002$, $.022, p=.02)$. In addition, while income as a control variable negatively predicted worry $(\beta=-.170, p<.001)$, it positively predicted a supportive attitude $(\beta=.118, p<.001)$.

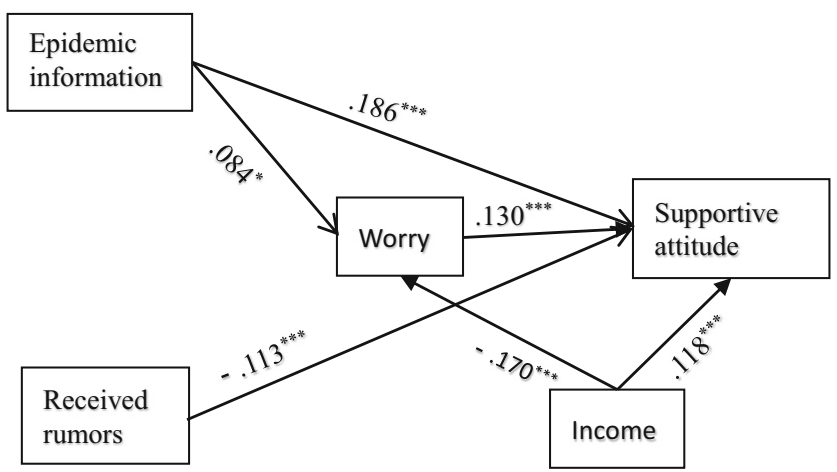

Fig. 2 The structure equation model of main variables note. The model shows associations between the amount of epidemic information, the amount of received rumors, worry and supportive attitude toward the epidemic prevention measures, controlling for the income of respondents. Coefficients presented are standardized linear regression coefficients. $* p<.05 . * * * p<.001$

\section{Discussion}

The present study investigated the impact of epidemic information and rumors on public's worries and attitude toward epidemic prevention measures among Chinese general population during the outbreak of the COVID-19 epidemic from the perspective of risk communication. Our results showed that epidemic information directly promoted public's supportive attitude toward epidemic prevention measures and indirectly via evoking a certain level of worry, while rumors among epidemic information damaged public's supportive attitude. These results can provide useful information for governments who are facing the challenge of epidemic outbreak to manage the public health crisis.

According to the results, the public's feelings of worry depended partially on the amount of epidemic information they acquired, thus confirming Hypothesis 1. Epidemic information informs public the real risk of infection and promotes risk perception. As an emotional response to perceived risk, the feeling of worry intensifies with an increase in epidemic information (Borkovec et al., 1998; Khosravi, 2020). Therefore, as noted previously, the public has exhibited a wide array of worries about the COVID-19 epidemic. However, while the influence of epidemic information on worry was significant, the effect size was small. It is possible that the highly saturated media market and comprehensive effective communication increase the public's exposure to information (Glik, 2007), ensuring almost everyone is aware of the epidemic risk. Faced with a highly infectious and low-fatal disease, the public may instinctively feel worried, once they are informed of this epidemic. This is not completely related to the amount of perceived information.

Our results demonstrated that the Chinese public exhibited a supportive attitude toward the epidemic prevention measures during the most serious period of the COVID-19 epidemic, and the more information individuals browsed through regarding the epidemic, the greater their supportive attitude was, thus supporting Hypothesis 2. Chinese public exhibited a high degree of cooperation, which is consistent with previous findings that public tend to take effective and adaptive collective actions during disasters when provided with useful information and coping strategies (Glik, 2007; Greenaway \& Cruwys, 2019). More importantly, the amount of epidemic 
information positively associated with public's supportive attitude, indicating that the function of risk communication was achieved by epidemic information to a certain degree. Good risk communication helps the public adapt to the dangerous environment and encourages them to take protective action as suggested (Vaughan \& Tinker, 2009). The Chinese government strived to enhance the public's awareness of prevention and intervention strategies by providing daily updates about surveillance and active cases on websites and social media. Many health workers, psychologists, and psychiatrists in China shared strategies through social media to protect the public's physical and mental health (Bao, Sun, Meng, Shi, \& Lin, 2020).

The mediation analysis revealed that worry positively predicted a supportive attitude toward epidemic prevention measures, and played a mediation role in the relationship between epidemic information and a supportive attitude. This supports Hypotheses 3 and 4. From the perspective of an attitude formation process, worry can motivate individuals to form a supportive explicit attitude toward epidemic prevention measures. This result is in line with the APE model of attitude formation. According to APE model, under a negative emotional state, individuals are more likely to adopt a propositional process (Wu et al., 2013), which can amend an implicit attitude to an explicit attitude more adaptive to the environment (Gawronski \& Bodenhausen, 2006, 2007). However, since this study didn't take implicit attitude into consideration, the relationship between implicit attitude, explicit attitude and emotional state during a public health crisis is remain to be uncovered in future researches. From a perspective of risk communication, worry can partially mediated the impact of epidemic information on the supportive attitude toward prevention measures, because the arousal of worry is essential in the process of risk communication wherein risk information motivates the public's protective behavior through evoking the feeling of worry, the emotional response to threat (Buck \& Ferrer, 2012). Therefore, unless the feeling of worry regarding the epidemic is aroused and the public perceives their life, health, and economy as threatened, they may find it difficult to support epidemic prevention measures. It is noteworthy that the indirect effect of worry in this study was very small. This may have been the result of the small effect size of epidemic information on the concern.

However, not every piece of epidemic information is conductive to risk communication. This study revealed that the more rumors people browsed through, the less supportive they became toward the prevention measures, which supports Hypothesis 5. In this study, the number of received rumors was indicated by the approximate proportion of browsed information that had been confirmed to be rumors. Thus, rumors did not only indicate inaccurate information but also inconsistent information. In risk communication, warnings can provide information for people at risk and enable them to take action, only when the warnings are credible and accurate (Glik, 2007; Sorensen, 2000). Rumors and inconsistent information reduce the credibility of the information, causing a failure of risk communication. Consequently, rumors related to COVID-19 reduced compliance with epidemic prevention measures (Tasnim et al., 2020).

Inconsistent with Hypothesis 6 and Hypothesis 7, based on the results, the number of received rumors were not correlated with worries. According to the literature, the effects of rumors on one's psychological state may vary across different categories of rumors (Bordia \& Difonzo, 2004). For example, dread rumors are related to how the virus spread and the range thereof, are likely to cause more severe worries, while wish rumors about new treatment methods and cures may reduce anxiety and even lead to an underestimate of the epidemic (Bordia \& Difonzo, 2004; Jiang, 2020). This may explain why the number of rumors did not predict the feeling of worry. It is recommended that future studies specifically examine the different categories.

Another interesting finding is that individuals with a low income experienced more worry during the COVID-19 epidemic and exhibit a more uncooperative attitude toward prevention measures. For those whose livelihoods are threatened, strict prevention measures increase negative emotions and the suspicion of official recommendations, thus affecting their willingness to comply with epidemic prevention measures adversely (Vaughan \& Tinker, 2009). Therefore, guaranteeing the basics to groups with a low income during COVID-19 is imperative to realize the full implementation of prevention measures.

\section{Conclusions and Implications}

To summarize, we found that during the COVID-19 epidemic (February 2020), the amount of epidemic information Chinese public received was positively associated with feeling of worry and supportive attitude toward the prevention measures. Worry played a partial mediation role in the impact of epidemic information on the supportive attitude. The amount of rumors was negatively associated with public's supportive attitude toward the prevention measures, but had no significant association with the feeling of worry. Besides, individuals with a low income experienced more worry and reported a less supportive attitude toward the prevention measures, highlighting the role of economic security for public health crisis management. The results of this study implied the importance of timely and credible information providing to evoke a certain level of worry and promote public supportive attitude, and the necessary attention to refute and resist rumors for effective risk communication in a public health crisis.

This study has important practical implications. First, it is necessary to maintain effective risk communication during the 
COVID-19 epidemic, as it can enhance the public's risk perception, and encourage the public to take officially recommended protective actions. Government and experts should provide the public with timely, accurate, and understandable information about the epidemiological characteristics of the outbreak, the scientific principles in the prevention measures, the curative effect of therapeutic activity, and the ways to reduce the risk of infection. Serious consequences of noncompliance with prevention measures should be communicated to the public in a direct and simple way to evoke a certain level of worry and increase their support for epidemic prevention measures. Second, attention should be paid to controlling the number of rumors and inconsistent information that emanate from various media platforms. Government should work with the media to communicate credible epidemic information to the public, and to enhance the monitoring of network media to reduce fake news and rumors. Furthermore, a lot of work should be done to refute rumors timely, so that the public can be reminded to resist rumors and refrain from spreading them. Third, attention should be paid to providing a living allowance and more guidance for groups with a low income to help alleviate excessively strong feelings of worry and to help them overcome this crisis.

\section{Limitations}

In this study, epidemic information browsing frequency and the number of rumors were measured by administering selfreport measures; thus, the assessment thereof may not have been objective enough. Because of the single-item measurement, this study just took the amount of epidemic information and rumors into consideration without other characteristics of information, such as type, source, and reliability, and couldn't investigate the impact of information detail. The sample was limited to non-infected people in the context of the Chinese culture. Therefore, the conclusions cannot be generalized to other groups. All the data were collected through an online survey, which implies that the respondents were primarily users of various social networking sites. Consequently, those who use the Internet and electronic devices less frequently could not be considered.

\footnotetext{
Authors' Contributions Conceived and designed the study: Hai-Ping Liao, Jin-Liang Wang.

Data collecting: Hai-Ping Liao.

Analyzed and discussed the data: Hai-Ping Liao, Jin-Liang Wang.

Wrote the original draft: Hai-Ping Liao.

Reviewed and edited the paper: Hai-Ping Liao, Jin-Liang Wang.
}

Funding This research was sponsored by Late-Stage Funding of Philosophy and Social Science Research Project by the Ministry of Education (grant number 20JHQ090).
Data Availability The data that support the findings of this study are available from the corresponding author upon reasonable request.

Code Availability Not applicable.

\section{Compliance with Ethical Standards}

Conflict of Interest On behalf of all authors, the corresponding author states that there is no conflict of interest.

Ethics Approval The study was reviewed and approved by the Ethics Committee of the Center for Mental Health Education, Southwest University.

\section{Appendices}

\section{The Results of EFA and CFA for The Epidemic Worry Scale}

To test the reliability and construct validity of The Epidemic Worry Scale, half of the valid data $(N=427)$ were randomly selected for an exploratory factor analysis (EFA), and the other half $(N=426)$ for a confirmatory factor analysis (CFA). The first half sample showed a good appropriateness of data for EFA, with $\chi^{2}=2259.964, d f=21, p<.001$ in the Bartlett's test of sphericity, and KMO (Kaiser-Meyer-Olkin) $=.894$. Common factor analysis with a maximum likelihood estimator was selected to extract latent factors, and then oblimin rotation was employed. Results showed that a single factor should be retained. All the communality estimates were larger than .468 , and factor loadings were ranging from .684 to .899 . The single factor named of Epidemic Worry accounted for $63.263 \%$ of the total variance. Cronbach's $\alpha$ was .925 in the first half sample. CFA for the other half sample revealed a good construct validity for this scale, $\chi^{2} / d f=3.117, p$ $=.001, \mathrm{CFI}=.992, \mathrm{TLI}=.981, \mathrm{RMSEA}=.071(95 \% \mathrm{CI}=.042$, $.101)$. Cronbach's $\alpha$ was .928 in the latter sample.

2. The Results of EFA and CFA for The Supportive Attitude toward Epidemic Prevention Measures Scale

The same way was employed to test the reliability and construct validity of this scale as the Epidemic Worry Scale. The first half sample $(N=427)$ showed a good appropriateness of data for EFA, with $\chi^{2}=888.570, d f=6, p<.001$ in the Bartlett's test of sphericity, and $\mathrm{KMO}=.703$. A single factor solution was retained, with communality estimates ranging from .285 to .834 , and factor loadings ranging from .534 to 913. The single factor named of Supportive Attitude accounted for $64.302 \%$ of the total variance. Cronbach's $\alpha$ was .783 in the first half sample. In the other half sample $(N$ $=426$ ), this scale had a good construct validity and reliability, $\chi^{2} / d f=3.016, p=.082, \mathrm{CFI}=.998, \mathrm{TLI}=.987, \mathrm{RMSEA}=$ .069 (95\% CI $=.000, .164)$, Cronbach's $\alpha=.793$. 


\section{References}

Ali, I. (2020). The COVID-19 pandemic: Making sense of rumor and fear. Medical Anthropology, 39(5), 376-379. https://doi.org/10. 1080/01459740.2020.1745481.

Bao, Y., Sun, Y., Meng, S., Shi, J., \& Lin, L. (2020). 2019-NCoV epidemic: Address mental health care to empower society. The Lancet, 6736(20), 2019-2020. https://doi.org/10.1016/S0140-6736(20) 30309-3.

Bish, A., \& Michie, S. (2010). Demographic and attitudinal determinants of protective Behaviours during a pandemic: A review. British Journal of Health Psychology, 15(4), 797-824. https://doi.org/10. 1348/135910710X485826.

Bordia, P., \& Difonzo, N. (2004). Problem solving in social interactions on the internet: Rumor as social cognition. Social Psychology Quarterly, 67(1), 33-49.

Borkovec, T. D., Ray, W. J., \& Stöber, J. (1998). Worry: A cognitive phenomenon intimately linked to affective, physiological, and interpersonal. Cognitive Therapy and Research, 22(6), 561-576. https:// doi.org/10.1023/A:1018790003416.

Brooks, S. K., Webster, R. K., Smith, L. E., Woodland, L., Wessely, S., Greenberg, N., \& Rubin, G. J. (2020). The psychological impact of quarantine and how to reduce it: Rapid review of the evidence. The Lancet, 395(10227), 912-920. https://doi.org/10.1016/S01406736(20)30460-8.

Buck R, and Ferrer, R. (2012). "Emotion, Warnings, and the Ethics of Risk Communication.” Pp. 640-717. In: Handbook of Risk Theory: Epistemology, Decision Theory, Tthics and Social Implications of Risk, edited by S. Roeser, R. Hillerbrand, P. Sandin, and M. Peterson. Springer.

Dong, M., \& Zheng, J. (2020). Letter to the editor: Headline stress disorder caused by Netnews during the outbreak of COVID-19. Health Expectations, 23(2), 259-260. https://doi.org/10.1111/hex.13055.

Freeston, M. H., Rhéaume, J., Letarte, H., Dugas, M. J., \& Ladouceur, R. (1994). Why do people worry? Personality and Individual Differences, 17(6), 791-802. https://doi.org/10.1016/01918869(94)90048-5.

Garrett, L. (2001). Understanding Media's Response to Epidemics. Public Health Reports, 116(Supplement 2), 67-91.

Gawronski, B., \& Bodenhausen, G. V. (2006). Associative and propositional processes in evaluation: An integrative review of implicit and explicit attitude change. Psychological Bulletin, 132(5), 692-731. https://doi.org/10.1037/0033-2909.132.5.692.

Gawronski, B., \& Bodenhausen, G. V. (2007). Unraveling the processes underlying evaluation: Attitudes from the perspective of the APE model. Social Cognition, 25(5), 687-717. https://doi.org/10.1521/ soco.2007.25.5.687.

Glik, D. C. (2007). Risk communication for public health emergencies. Annual Review of Public Health, 28, 33-54. https://doi.org/10.1146/ annurev.publhealth.28.021406.144123.

Greenaway, K. H., \& Cruwys, T. (2019). The source model of group threat: Responding to internal and external threats. American Psychologist, 74(2), 218-231. https://doi.org/10.1037/ amp0000321.

Jalali, R., \& Mohammadi, M. (2020). Rumors and incorrect reports are more deadly than the new coronavirus (SARS-CoV-2). Antimicrobial Resistance and Infection Control, 9, 68. https://doi. org/10.1186/s13756-020-00738-1.

Jiang, S. (2020). Information epidemic: The spreading of rumor about COVID-19 and the coping strategies. Studies on Science Popularization(in Chinese), 1, 70-78.

Khosravi, M. (2020). Perceived risk of COVID-19 pandemic: the role of public worry and trust. Electronic Journal of General Medicine, 17(4), em203. https://doi.org/10.29333/ejgm/7856.
Kummeneje, A. M., \& Rundmo, T. (2019). Risk perception, worry, and pedestrian behaviour in the Norwegian population. Accident Analysis and Prevention, 133, 105294. https://doi.org/10.1016/j. aap.2019.105294.

MacKinnon, D. P., Fairchild, A. J., \& Fritz, M. S. (2007). Mediation analysis. Annual Review of Psychology, 58, 593-614. https://doi. org/10.2307/3412968.

Mogg, K., \& Bradley, B. P. (2004). A cognitive-motivational perspective on the processing of threat information and anxiety. In J. Yiend (Ed.), Cognition, emotion and psychopathology (pp. 68-85). Cambridge: Cambridge University Press.

Olagoke, A. A., Olagoke, O. O., \& Hughes, A. M. (2020). Exposure to coronavirus news on mainstream media: The role of risk perceptions and depression. British Journal of Health Psychology, 25, 1-10. https://doi.org/10.1111/bjhp.12427.

Parikh, P. A., Shah, B. V., Phatak, A. G., Vadnerkar, A. C., Uttekar, S., Thacker, N., \& Nimbalkar, S. M. (2020). COVID-19 pandemic: Knowledge and perceptions of the public and healthcare professionals. Cureus, 12(5), e8144. https://doi.org/10.7759/cureus.8144.

Podsakoff, P. M., \& Organ, D. W. (1986). Self-reports in organizational research: Problems and prospects. Journal of Management, 12(4), 531-544. https://doi.org/10.1177/014920638601200408.

Rosnow, R. L. (1980). Psychology of rumor reconsidered. Psychological Bulletin, 87(3), 578-591. https://doi.org/10.1037/0033-2909.87.3. 578.

Sibley, C. G., Greaves, L. M., Satherley, N., Wilson, M. S., Overall, N. C., Lee, C. H. J., Milojev, P., Bulbulia, J., Osborne, D., Milfont, T. L., Houkamau, C. A., Duck, I. M., Vickers-Jones, R., \& Barlow, F. K. (2020). Effects of the COVID-19 pandemic and Nationwide lockdown on trust, attitudes toward government, and well-being. American Psychologist, 75(5), 618-630. https://doi.org/10.1037/ amp0000662.

Smith, R. D. (2006). Responding to global infectious disease outbreaks: Lessons from SARS on the role of risk perception, communication and management. Social Science and Medicine, 63(12), 3113-3123. https://doi.org/10.1016/j.socscimed.2006.08.004.

Sorensen, J. H. (2000). Hazard warning systems: Review of 20 years of Progress. Natural Hazards Review, 1(2), 119-125.

Tasnim, S., Hossain, M. M., \& Mazumder, H. (2020). Impact of Rumors or Misinformation on Coronavirus Disease (COVID-19) in social media. Journal of Preventive Medicine and Public Health, 53(3), 171-174. https://doi.org/10.31235/osf.io/uf3zn.

Vaughan, E., \& Tinker, T. (2009). Effective health risk communication about pandemic influenza for vulnerable populations. American Journal of Public Health, 99(SUPPL. 2), 324-332. https://doi.org/ 10.2105/AJPH.2009.162537.

Wallace, D. S., Paulson, R. M., Lord, C. G., \& Bond, C. F. (2005). Which behaviors do attitudes predict? Meta-analyzing the effects of social pressure and perceived difficulty. Review of General Psychology, 9(3), 214-227. https://doi.org/10.1037/1089-2680.9.3.214.

Wu, M., Fang, X., Sun, X., \& Li, Q. (2013). Emotion, attitude and behavior:From perspective of APE model. Journal of Psychological Science, 36(3), 706-710.

Yamashita. (2012). A review of psychosocial assessments for disaster mental health studies. Psychological Trauma Theory Research Practice and Policy, 4(6), 560-567. https://doi.org/10.1037/ a0025952.

Publisher's Note Springer Nature remains neutral with regard to jurisdictional claims in published maps and institutional affiliations.

Publisher's NotePublisher's Note Springer Nature remains neutral with regard to jurisdictional claims in published maps and institutional affiliations. 